\section{Inconsistency between Danish incidence and prevalence data about psoriatic arthritis (PsA)}

We are grateful to Egeberg and Kristensen for presenting the detailed data about the prevalence and incidence of psoriatic arthritis (PsA). ${ }^{1}$ Based on these detailed information, we tried to estimate the excess mortality of people with diagnosed PsA by uising a mathematical relation between incidence, prevalence and mortality. ${ }^{23}$ During analysis of the incidence and prevalence data, we have made the following observation: if we assume that-on population average-people with PsA do not have a better survival than those without PsA, we can compute a lower bound for the incidence rate from the prevalence data (the details for the derivation of the lower bound can be found in the Appendix). We calculated this mathematical lower bound based on the prevalence data and compared the lower bound with the incidence data given in Ref 1 . We found that in less than $50 \%$ of the strata where incidence data were given, the corresponding mathematical lower bounds have been reached (or exceeded). For instance, the lower bound for the incidence rate in the age group 40-49 in 2009 is 43.3 per 100000 person-years (both sexes). The observed incidence rate in this stratum is only 29.8 per 100000 person-years-a deviation of more than 30\%. More than half of the reported incidence rates stratified by age and year are implausibly small given the observed prevalence values. Unfortunately, we do not have an explanation for the inconsistencies between the incidence and prevalence data. Possibly, in estimating the age-specific prevalence, some double counting of cases has occurred.

Appendix: Deriving a lower bound for the age-specific incidence rate

Mathematically, it can be shown that

$$
\partial \mathrm{p}=(1-\mathrm{p}) \times\{\mathrm{i}-\mathrm{p} \times(\mathrm{m} 1-\mathrm{m} 0)\}
$$

where $\partial p$ is the temporal change of the age-specific prevalence $\mathrm{p}$ with respect to time and age. ${ }^{12}$ The rates $\mathrm{i}, \mathrm{m} 0$ and $\mathrm{m} 1$ are the age-specific incidence and mortality rates of the people with (m1) and without diagnosed PsA (m0).

A straightforward calculation yields that

$$
\partial \mathrm{p} /(1-\mathrm{p})+\mathrm{p} \times(\mathrm{m} 1-\mathrm{m} 0)=\mathrm{i} .
$$

With the assumption that the mortality rate of the people without PsA is not higher than the mortality of the people with PsA, that is, $\mathrm{m} 0 \leq \mathrm{m} 1$, this equation implies

$$
\partial \mathrm{p} /(1-\mathrm{p}) \leq \mathrm{i}
$$

This means the incidence rate (i) is always greater than the temporal change $(\partial p)$ of the prevalence over 1 minus the age-specific prevalence. Thus, we have a lower bound for the incidence rate.

The question arises, if the assumption $\mathrm{m} 0 \leq \mathrm{m} 1$ is reasonable on the population level (here: Denmark). The main reason for this assumption being true (on the population level) is that PsA often is a severe disease coming along with severe side effects and disease-specific complications. Hence, it appears reasonable to us that equation (1) yields a lower bound for the incidence rate.

Philipp Sewerin, ${ }^{1}$ Annika Hoyer, ${ }^{2}$ Matthias Schneider, ${ }^{1}$ Benedikt Ostendorf, ${ }^{1}$ Ralph Brinks ${ }^{1}$

${ }^{1}$ Department and Hiller Research Unit of Rheumatology, University Düsseldorf, Düsseldorf, Germany

${ }^{2}$ Institute for Biometry and Epidemiology, German Diabetes Center, Düsseldorf, Germany

Correspondence to Dr Philipp Sewerin, Department and Hiller Research Unit of Rheumatology, University Duesseldorf, Duesseldorf 40225, Germany; philipp.sewerin@med.uni-duesseldorf.de

Handling editor Josef S Smolen

Competing interests None declared.

Provenance and peer review Not commissioned; internally peer reviewed.

(c) Article author(s) (or their employer(s) unless otherwise stated in the text of the article) 2019. All rights reserved. No commercial use is permitted unless otherwise expressly granted.

\section{Check for updates}

To cite Sewerin P, Hoyer A, Schneider M, et al. Ann Rheum Dis 2019;78:e44.

Received 8 December 2017

Accepted 15 December 2017

Published Online First 2 January 2018

Ann Rheum Dis 2019;78:e44. doi:10.1136/annrheumdis-2017-212817

\section{REFERENCES}

1 Egeberg A, Kristensen LE. Impact of age and sex on the incidence and prevalence of psoriatic arthritis. Ann Rheum Dis 2017.

2 Brinks R, Landwehr S. Age- and time-dependent model of the prevalence of noncommunicable diseases and application to dementia in Germany. Theor Popul Biol 2014;92:62-8.

3 Brinks R, Landwehr S. A new relation between prevalence and incidence of a chronic disease. Math Med Biol 2015;35:425-35. 